\title{
Inter- and Intramolecular Interactions of Polymer as Studied by Fluorescence Spectroscopy XVI. Synthesis and Exciplex Emission Properties of Poly[oxy-2,2-(1-pyrenylmethyl) (4- $N, N$-dimethylaminobenzyl)propyleneoxycarbonyl- iminohexamethyleneiminocarbonyl]
}

\author{
Hui Liang YUAN and Shigeo TAZUKE \\ Research Laboratory of Resources Utilization, \\ Tokyo Institute of Technology, \\ 2459 Nagatsuta, Midori-ku, Yokohama, Japan
}

(Received July 5, 1982)

\begin{abstract}
An exciplex forming polyurethane (I) $(n \leq 17)$, its reference model polyurethane (III), and the monomer model (II) were prepared. The catalyst effects on the synthesis of polyurethane are discussed. From the results of absorption, emission and excitation spectra, the concentration and molecular weight effects on inter- and intramolecular interactions of $\mathbf{I}$ in 1,2dichloroethane were compared with those of II and III. The ratio of exciplex emission intensity $\left(F_{\mathrm{e}}\right)$ to monomer emission intensity by pyrenyl groups $\left(F_{\mathrm{m}}\right)$ increased with concentration in an intermediate DP region, suggesting the interpolymer association of $\mathbf{I}$ in dilute solutions. No such tendency was observed for II and III. Clear differences in I and the analogous polyester IV were found. The maximum broadness of absorption spectra was observed in a medium DP region for I but not for IV. The maximum interpolymer association of I occurred in the lower DP region in contrast to IV. The poor solubility of the polyurethane brought about by hydrogen bonding and the resulting rigid main chain probably account for these differences. The weaker exciplex forming tendency of I than IV was attributed to the participation of DMA in the hydrogen bonding. This was supported by infrared and NMR spectroscopy.

KEY WORDS Exciplex / Electron Donor Acceptor / Hydrogen Bonding /

Polyurethane / Fluorescence / Pyrene / N,N-Dimethylaniline / Polymer

Interaction / Molecular Association / Polyaddition /
\end{abstract}

In the preceding article, we have discussed the exciplex emission of 1-(1-pyrenyl)-3-(4- $N, N$-dimethylanilino)propane group bonded to a polyester. $^{1}$ One advantage of employing the pyrene (Py)-N,N-dimethylaniline (DMA) pair as a chromophore is the variety of choice of polymer structure. The aim of this article is to study the effect of a polymer main chain structure on intra- and interpolymer interactions. We chose polymer $\mathbf{I}$ and its monomer model compound II and the reference polymer III and compared the fluorescence and absorption characteristics with those of IV and $\mathbf{V}$ reported in the preceding contributions. ${ }^{1,2}$ The polymers I and IV are isostructural except for a small difference from substituting two methylene groups in the main chain of IV with two imino groups in I. The space between the chromophore pairs is consequently nearly the same but the introduction of hydrogen bonding groups is expected to affect the features of the inter- and intrapolymer interactions.

When urethane bonds are introduced into a polymer chain, the polymer chain becomes stiff and the segment mobility of either the main or side chains is greatly reduced. ${ }^{3,4}$ We have demonstrated the important role of hydrogen bonding urethane groups in controlling the photodimerization of anthryl groups either bonded as polymer side groups or incorporated in a polymer main chain. In comparison with the relevant polyesters, segment diffusion to a paired location suitable for photodimerization is hindered whereas the paired chro- 
mophores once formed remain undisturbed in polyurethane. Although fluorescence and absorption spectroscopy of the anthryl groups provided information on the segment mobility, more detailed and intensive information can be obtained from the study on an exciplex sensitive to small changes in environments.

In the following, we are going to discuss the intraand interpolymer interactions relevant to very weak electron donor acceptor (EDA) interaction. Since hydrogen bonding is essentially an acid-base interaction with a small contribution of a charge transfer nature, ${ }^{5}$ hydrogen bonding and the weak EDA interaction between Py and DMA might cooperate or cancel out each other.

\section{EXPERIMENTAL}

\section{Materials}

Poly[oxy-2,2-(1-pyrenylmethyl)(4-N,N-dimethylaminobenzyl) propyleneoxycarbonyliminohexamethyleneiminocarbonyl] (I). An anhydrous anisole solution $(10 \mathrm{ml})$ containing $0.88 \mathrm{~g}(2.30 \mathrm{mmol})$ of 2-(4- $N, N$-dimethylaminobenzyl)-2-(1-pyrenylmethyl)propane-1,3-diol and di- $n$-butyltin dilaurate and triethylamine $(0.1 \mathrm{wt} \%$ of the diol) as catalyst was stirred at $150-160^{\circ} \mathrm{C}$. Anhydrous anisole solution $(4 \mathrm{ml})$ containing $0.4 \mathrm{~g}(2.30 \mathrm{mmol})$ of hexamethylene diisocyanate was added in two portions, the second following $5 \mathrm{~h}$ after the first, by a dropping funnel. If all the isocyanate was added at once, gel formation took place. The reaction mixture was refluxed with stirring for $7 \mathrm{~h}$. The polymer was precipitated in methanol. Poly[oxy-2,2-(1-pyrenylmethyl) (benzyl)propyleneoxycarbonyliminohexamethyleneiminocarbonyl] (III) was synthesized by a method similar to that described above. Analytical data for I: E.A. Calcd for $\mathrm{C}_{37} \mathrm{H}_{41} \mathrm{O}_{4} \mathrm{~N}_{3}$ (repeating unit); C, 75.10\%; H, 6.98\%; N, 7.09\%. Found: C, $76.10 \%$; H, 7.01\%; N, 6.85\%. IR (KBr disk) $3420,3330\left(v_{\mathrm{NH}}\right), 1700 \mathrm{~cm}^{1}\left(v_{\mathrm{C}=\mathrm{o}}\right)$. NMR $\left(\mathrm{CDCl}_{3}\right) \delta 0.80-1.55\left(8 \mathrm{H}, \mathrm{m},-\left(\mathrm{CH}_{2}\right)_{4}\right), 2.91(6 \mathrm{H}$, $\left.\mathrm{s}, \quad-\mathrm{N}\left(\mathrm{CH}_{3}\right)_{2}\right), \quad 3.38-3.68 \quad\left(2 \mathrm{H}, \quad \mathrm{m}, \quad-\mathrm{CH}_{2}-\mathrm{Py}\right)$, $3.81-4.26\left(4 \mathrm{H}, \mathrm{m},-\mathrm{OCH}_{2}-\right), 4.60-5.10(2 \mathrm{H}, \mathrm{m}$, -NH-), $6.50-7.21$ (4H, m, phenyl), and $7.61-8.24$ (9H, m, pyrenyl).

Analytical data for III: E.A. Calcd for $\mathrm{C}_{35} \mathrm{H}_{36} \mathrm{O}_{4} \mathrm{NO}_{2}$ (repeating unit); $\mathrm{C}, 76.62 \% ; \mathrm{H}$, $6.64 \%$; N, 5.10\%. Found: C, 76.37\%; H, 6.98\%; N, $5.30 \%$ IR (KBr disk) 3420, $3320\left(v_{\mathrm{NH}}\right), 1720 \mathrm{~cm}^{-1}$
$\left(v_{\mathrm{C}=\mathrm{o}}\right)$

1,3-Bis( $N$-butylcarbamoyloxy)-2-(1-pyrenylmethyl)-2-(4-N,N-dimethylaminobenzyl)propane (II). To an anhydrous anisole solution $(10 \mathrm{ml})$ containing $0.65 \mathrm{~g}(1.54 \mathrm{mmol})$ of 2-(4- $N, N$-dimethyl aminobenzyl)-2-(1-pyrenylmethyl) propane-1,3-diol was added an anhydrous anisole solution $(5 \mathrm{ml})$ containing $0.7 \mathrm{~g}(3.10 \mathrm{mmol})$ of $n$-butyl isocyanate and a catalytic amount of di- $n$-butyltin dilaurate at $150-160^{\circ} \mathrm{C}$ under stirring. After $6 \mathrm{~h}$, the solvent was removed by distillation under reduced pressure. The product was purified on a silica gel column with benzene as the eluent. Yield, $0.86 \mathrm{~g}(91.5 \%) \mathrm{mp}$ $166-167^{\circ} \mathrm{C}$. Analytical data: E.A. Calcd for $\mathrm{C}_{39} \mathrm{H}_{47} \mathrm{O}_{4} \mathrm{~N}_{3}$ : C, $75.33 \%$; $\mathrm{H}, 7.60 \%$; N, $6.75 \%$. Found: C, 75.15\%; H, 7.65\%; N, 6.61\%. IR (KBr disk) 3420 (shoulder), $3320\left(v_{\mathrm{NH}}\right), 1720\left(v_{\mathrm{C}=\mathrm{O}}\right) \mathrm{cm}^{-1}$. NMR $\left(\mathrm{CDCl}_{3}\right) \delta 0.72-1.00\left(6 \mathrm{H}, \mathrm{t},-\mathrm{CH}_{3}\right), 1.00-$ $1.55\left(8 \mathrm{H}, \mathrm{m},-\mathrm{CH}_{2} \mathrm{CH}_{2}-\mathrm{Me}\right), 2.81\left(2 \mathrm{H}, \mathrm{s},-\mathrm{CH}_{2}-\right.$ DMA), $2.95\left(6 \mathrm{H}, \mathrm{s},-\mathrm{N}\left(\mathrm{CH}_{3}\right)_{2}\right), 2.95-3.21(4 \mathrm{H}, \mathrm{m}$, $\left.-\mathrm{NHCH}_{2}-\right), 3.55\left(2 \mathrm{H}, \mathrm{s},-\mathrm{CH}_{2}-\mathrm{Py}\right), 4.00(4 \mathrm{H}, \mathrm{s}$, $\left.-\mathrm{OCH}_{2}-\right), 4.10-4.60(2 \mathrm{H}, \mathrm{m},-\mathrm{NH}), 6.60-7.18(4 \mathrm{H}$, $\mathrm{m}$, phenyl), and 7.78-8.21 (9H, m, Py).

The polyurethane sample was fractionated by gel permeation chromatography (HLC-802UR, Toyo Soda Mfg. Co. Ltd., columns for fractionation: $\mathrm{G}$ $2000 \mathrm{HG}_{6}+\mathrm{G} 3000 \mathrm{HG}_{6}$, columns for molecular weight measurement: $\mathrm{GMH} \times 2+\mathrm{G} \quad 4000 \mathrm{H}+\mathrm{G}$ $2500 \mathrm{H}$, eluent : chloroform, operation temperature: $35^{\circ} \mathrm{C}$ ).

\section{Solvent}

Spectrograde 1,2-dichloroethane was further purified by distillation before use. Other solvents were purified by accepted procedures.

\section{Spectroscopy}

IR spectra were recorded on a Hitachi 260-10 type infrared spectrometer. All samples were measured in the solid state; I and III were cast on a $\mathrm{NaCl}$ plate from chloroform solutions while II was measured as a $\mathrm{KBr}$ disk. The absorption and fluorescence spectra were recorded on a Shimadzu UV-200 spectrometer and a Hitachi MPF-4 spectrofluorometer, under an argon atmosphere and no correction was made on for the fluorescence spectra. The molarity of the polymer solution was based on the "repeating unit" concentration. The NMR spectra were recorded on a $100 \mathrm{MHz}$ (JEOL) JNMPS-100 spectrometer. The exciplex emission quan- 
tum yield was measured as already reported. ${ }^{6}$

\section{RESULTS AND DISCUSSION}

\section{Polymer Synthesis}

To investigate the effects of the polymer main chain structure on inter- and intramolecular exci- plex formation, the following polymers and the corresponding monomer model were designed. Polyurethanes I and III were prepared from the corresponding diol monomers and hexamethylene diisocyanate. For the preparation of polyurethanes, anisole or chlorobenzene commonly used as the solvent. However, the halogenated solvent was not

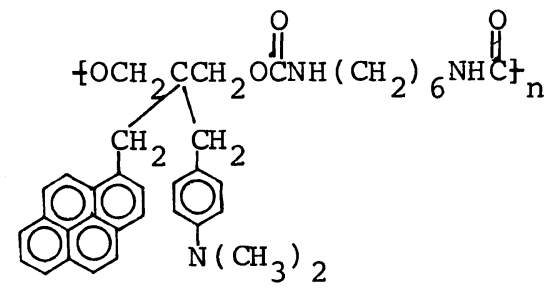

I

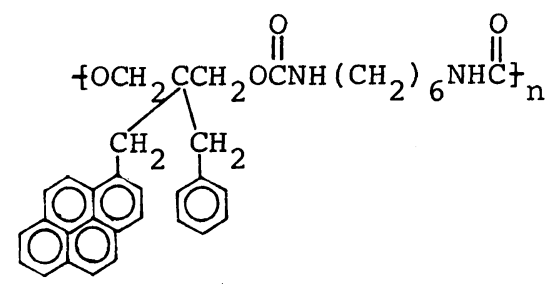

III

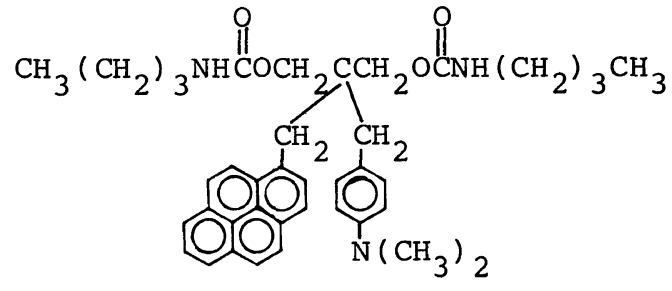

II<smiles>CC(=O)CC(Cc1ccc2ccccc2c1)CC(CO)Cc1ccc(N(C)C)cc1</smiles>

IV<smiles>CC(=O)OCCC(Cc1ccc(CC(CCN(C)C)C(C)=O)cc1)Cc1ccc2ccccc2c1</smiles>

V

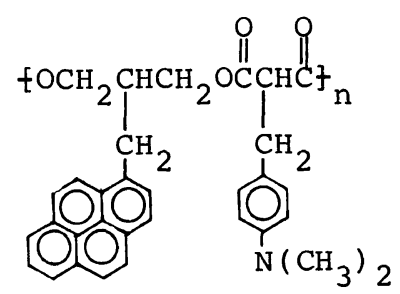

VI

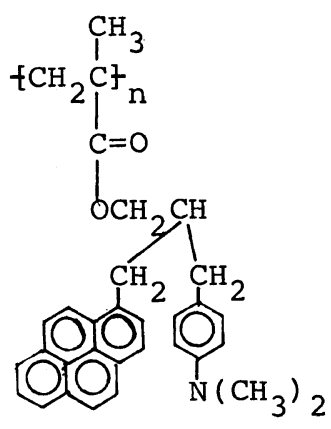

VII

Scheme 1. 
Table I.

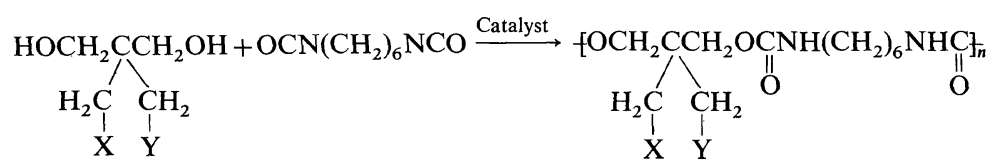

\begin{tabular}{cllcrr}
\hline Exptl no. & $\mathrm{X}$ & $\mathrm{Y}$ & Catalyst $^{\mathrm{a}}$ & \multicolumn{2}{c}{$M_{w}{ }^{\mathrm{b}}$} \\
\hline 1 & Py & DMA & None & \multicolumn{2}{c}{ No polymer } \\
2 & Py & DMA & $(1)$ & 2000 & 3.5 \\
3 & Py & DMA & $(1)+(2)$ & 10000 & 17.0 \\
4 & Py & Ph & None & \multicolumn{2}{c}{ No polymer } \\
5 & Py & Ph & $(1)$ & 2000 & 3.5 \\
6 & Py & Ph & $(1)+(2)$ & 2000 & 3.5 \\
\hline
\end{tabular}

a Catalyst (1) $\left[\mathrm{CH}_{3}\left(\mathrm{CH}_{2}\right)_{3}\right]_{2} \mathrm{Sn}\left[\mathrm{OOC}\left(\mathrm{CH}_{2}\right)_{10} \mathrm{CH}_{3}\right]_{2} ;(2)\left(\mathrm{C}_{2} \mathrm{H}_{5}\right)_{3} \mathrm{~N}$.

b Estimated for unfractionated polymers by GPC; reference: standard polystyrenes.

suitable for high temperature soluion polymerization of the present diol containing amine. Addition polymerization in anisole gives an almost colorless polyurethane favorable for spectroscopic study. The molar ratio of diol/diisocyanate was strictly maintained at 1 in order to obtain a high molecular weight polymer. The results and the preparative procedure of the polyurethanes are shown in Table I. As shown in Table I (\#1, \#4), no polyurethanes were obtained when the catalyst was absent. The catalyst effects on the degree of polymerization (DP) of the resulting polyurethane were dependent very much on the presence of amino groups in the diol monomer. The poor reactivity of 2-(1-pyrenyl)-2-benzyl-1,3-propanediol (\#5, \#6) even in the presence of catalysts may be attributed to the poor solubility of III. When the polymer precipitates, further propagation is no longer possible. The better solubility of $\mathbf{I}$ is probably one reason for obtaining relatively high DP polymers (\#3). However, this is possible only when both di- $n$ butyltin dilaulate and triethylamine are used (compare \#2 and \#3). If the tin compound is coordinated to DMA group, the catalyst may lose its activity. In this case, destruction of the coordination bond by the addition of a stronger base (triethylamine) would be required to restore the catalytic activity. The catalyst effects in the polyurethane formation are very complicated. Another explanation is that a ternary complex may be formed among the diol monomer, the tin compound and triethylamine, which might accelerate the addition polymerization. $^{7}$

\section{Absorption Spectroscopy}

The absorption spectra of I and II are shown in Figure 1. Although the peaking wavelengths of the absorption spectrum of $\mathbf{I}$ in DCE are nearly identical with those of II, the broadening of the spectrum of I expressed by the valley/peak ratio (i.e., $\mathrm{OD}_{335} /$ $\mathrm{OD}_{346}=0.39-0.48$ ) is more prominent than that of either II $\left(\mathrm{OD}_{335} / \mathrm{OD}_{346}=0.30\right)$ or III $\left(\mathrm{OD}_{335} /\right.$ $\mathrm{OD}_{346}=0.32$ ). It seems to be a common fact that the absorption spectra of an exciplex forming polymer are broader than those of relevent low mol wt model compounds and excimer forming polymers. ${ }^{8-11}$ Comparison of I with IV indicates that the absorption spectra of the Py-DMA pair are affected considerably by a small difference in the polymer main chain. The absorption spectra of $\mathbf{I}$ are broader than the analogous polyester IV. Since the separation between chromophores is identical for I and IV, the clear difference in the absorption spectra $\left(\mathrm{OD}_{335} / \mathrm{OD}_{346}=0.39-0.48\right.$ for $\mathbf{I}$, and 0.33 0.36 for IV) results from the rigid main chain of $\mathbf{I}$. Our previous finding that the absorption spectra of IV are broader in a good solvent (DCE) than in a poor solvent (mesitylene) suggests an extended polymer chain to be favorable to EDA interaction. By analogy, the broader absorption spectrum of I is attributable to its rigid, and therefore, extended polyurethane main chain. Since the relevent monomer models, II and $\mathbf{V}$, exhibit almost identical absorption spectra, the effect of the urethane group is specific for the polymer system.

The broadness of absorption spectra of $\mathbf{I}$ is plotted against DP as shown in Figure 2. It is 


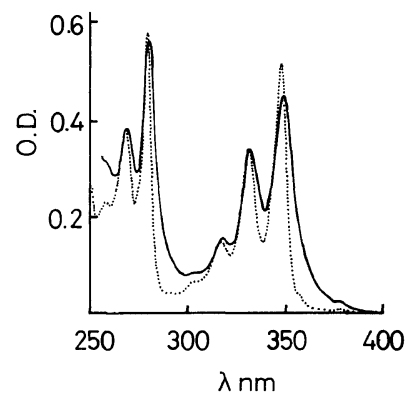

Figure 1. Absorption spectra of $\mathbf{I}$ and $\mathbf{I}$ in DCE. $[\mathrm{Py}]=2 \times 10^{-5}$ M. I, (-); II, (-----).

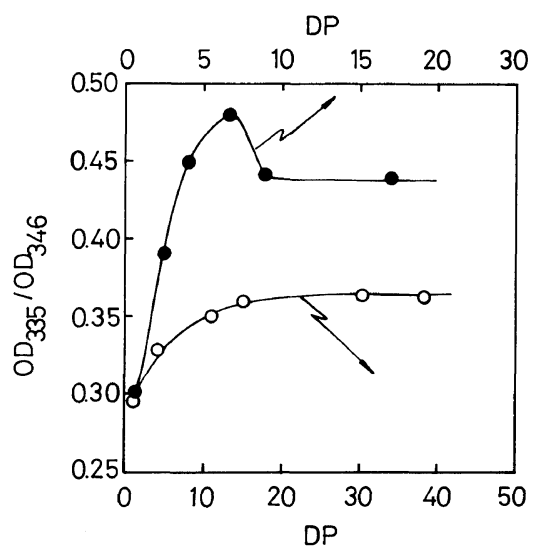

Figure 2. Comparison of I with IV in DP dependent broadening of the absorption spectra in DCE. [Py]= $2 \times 10^{-5}$ M. I, (O); IV, (O).

interesting to note that the maximum broadness of absorption spectra of I was found for a medium sized polyurethane. For IV, the absorption spectra become broader with increasing DP whereas the origin of the broadening is the intrapolymer interaction as indicated by obedience to the Beer's law. The fraction 5 of $\mathbf{I}$ is very specific, as will be discussed in the following section, and many physical values show the maximum value in this DP region.

\section{Fluorescence Spectroscopy}

The fluorescence spectra of I, II, and III are shown in Figure 3 and the emission characteristics are shown in Table II along with the reported results on IV. ${ }^{1,2}$ The maximum wavelength of the exciplex emission by $\mathbf{I}$ depends on DP as plotted in Figure 4. The complexed shape of the plots at $c=$

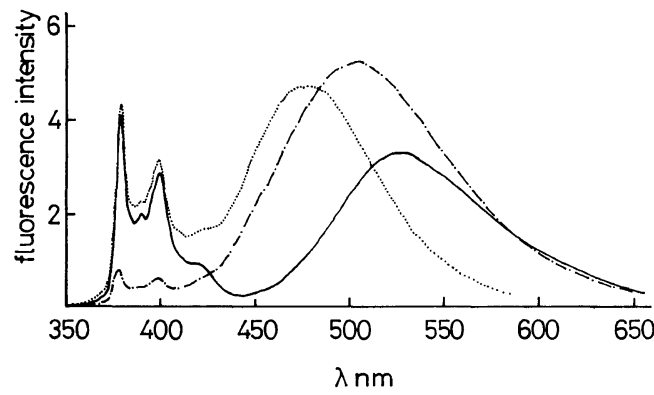

Figure 3. Fluorescence spectra of I (Fraction 5), II, and III in DCE. Excitation at $346 \mathrm{~nm},[\mathrm{Py}]=5 \times 10^{-5} \mathrm{M}$. I, (----); II, (- - ; III, (-----).

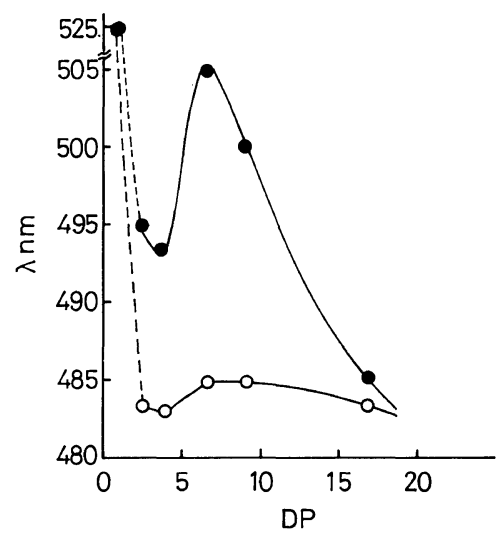

Figure 4. DP dependence of the maximum exciplex emission wavelength of $I$ in DCE. Excitation at $346 \mathrm{~nm}$. $[\mathrm{Py}]=5 \times 10^{-5} \mathrm{M}(\bigcirc)$ and $<1 \times 10^{-6} \mathrm{M}(\mathrm{O})$.

$5 \times 10^{-5} \mathrm{M}$ seems to be a reflection of mixed interand intrapolymer exciplexes as well as excited EDA complexes. The contribution of each component depends on DP and the concentration of $\mathbf{I}$. Comparing Figure 4 with Figure 7 and Table II, it can be seen that the open circles in Figure 4 represent the intrapolymer exciplex and possibly the EDA complex almost exclusively whereas the black circles include both the inter- and intrapolymer exciplexes. In particular, the fractions 3 and 5 are prone to the interpolymer exciplex formation so that the wavelengths of peaking emission are practically of the interpolymer exciplex. The results in Figure 4 are contrasting to those in Figure 10 in the previous report. ${ }^{1}$ For IV, the inter- and intrapolymer exciplexes appeared at the same wavelength whereas the interpolymer exciplex by $\mathbf{I}$ emits in a longer wavelength region than the intrapolymer 
Table II.

\begin{tabular}{|c|c|c|c|c|c|c|c|c|}
\hline \multirow{3}{*}{ Sample } & \multirow{3}{*}{ No. } & \multirow{3}{*}{$M_{w}{ }^{a}$} & \multirow{3}{*}{$\mathrm{DP}$} & \multirow{3}{*}{$\begin{array}{c}\text { Broadness }^{\mathrm{b}} \\
\text { of absorption } \\
\text { spectra }\end{array}$} & \multicolumn{4}{|c|}{ Fluorescence spectra } \\
\hline & & & & & $\lambda_{\mathrm{m}}^{\max } \mathrm{c}$ & $\lambda_{\mathrm{e}}^{\max c}$ & $\lambda_{\mathrm{e}}^{\max }{ }_{c \rightarrow 0}{ }^{\mathrm{d}}$ & \\
\hline & & & & & $\mathrm{nm}$ & $\mathrm{nm}$ & $\mathrm{nm}$ & \\
\hline \multirow{5}{*}{ I } & 1 & 10500 & 17.0 & 0.44 & 377,398 & 485 & 483 & 0.5 \\
\hline & 3 & 5500 & 9.0 & 0.44 & 377,398 & 500 & 485 & $0.6 \pm 0.3$ \\
\hline & 5 & 3900 & 6.5 & 0.48 & 377,398 & 505 & 485 & $0.5 \pm 0.3$ \\
\hline & 7 & 2000 & 3.5 & 0.45 & 377,398 & 493 & 482 & 0.5 \\
\hline & 9 & 1600 & 2.5 & 0.39 & 377,398 & 495 & 483 & 0.5 \\
\hline II & $\begin{array}{l}\text { Monomer } \\
\text { model }\end{array}$ & 621.8 & 1 & 0.30 & 377, 398 & 525 & 525 & 0.5 \\
\hline III & $\begin{array}{l}\text { Reference } \\
\text { polymer }\end{array}$ & $2000^{\mathrm{e}}$ & 3.5 & 0.32 & 377,398 & 476 & 478 & $\begin{array}{c}1.1 \\
\text { (excimer) }\end{array}$ \\
\hline IV & Polyester & 17800 & 30.0 & 0.36 & 377,398 & 515 & 482 & 4.0 \\
\hline $\mathbf{V}$ & $\begin{array}{l}\text { Monomer } \\
\text { model of IV }\end{array}$ & 507.4 & 1 & 0.28 & 377,398 & 525 & 525 & 2.2 \\
\hline
\end{tabular}

a Determined by GPC (reference: polystyrene).

b $\mathrm{OD}_{335} / \mathrm{OD}_{346}$ (valley/peak ratio).

c Pyrene monomer and exciplex emission maximum wavelength at $[\mathrm{Py}]=5 \times 10^{-5} \mathrm{M}$.

d Maximum wavelength of exciplex (excimer) emission at the high dilution limit.

e Unfractionated sample. The $F_{\mathrm{e}} / F_{\mathrm{m}}$ value is independent of concentration.

exciplex. The contribution of EDA emission seems rather small judging from the weak EDA emission even in a frozen matrix (Figure 5). The lower energy emission by II in comparison with $\mathbf{I}$ is comparable to the same relation between IV and $\mathbf{V}$. Reduced solvation and steric hindrance in polymers accounts for this.

It is of interest to recognize the difference in $\lambda_{\mathrm{e}}^{\max }$ (Table II) between I and IV. Since the monomer models II and $\mathbf{V}$ emit an identical exciplex emission, the higher exciplex energy of I than IV may be attributed to hydrogen bonding which reduces the electron donor properties of DMA. Hydrogen bonding also brings about a rigid main chain so that the relaxation of the exciplex is retarded, giving rise to a blue shifted exciplex emission.

The ground state interaction is shown in Figures 5 and 6 . In a glass matrix at $77 \mathrm{~K}$ (Figure 5), the formation of a "true" exciplex is prohibited and the remaining broad emission extending over $500 \mathrm{~nm}$ is considered as due to an excited EDA complex. The EDA emission was observed more or less for all polymers containing Py and DMA groups. Particularly, VI and VII in Scheme 1 exhibited strong emission peaking around $440 \mathrm{~nm} .{ }^{9}$ II or III hardly exhibited the EDA emission. The excitation

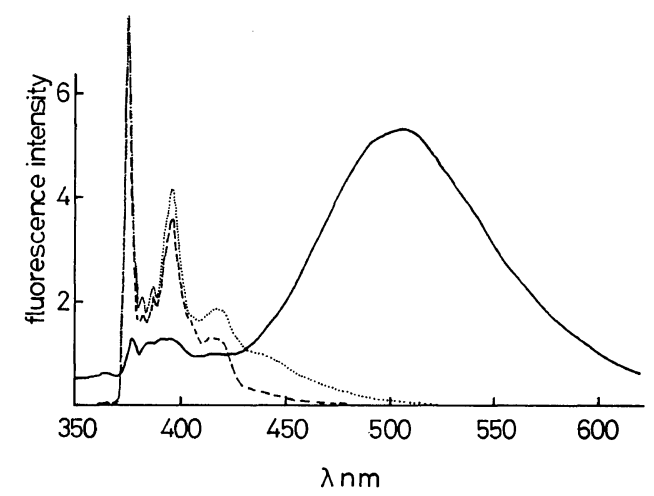

Figure 5. Fluorescence spectra in a glassy MTHF matrix. $[\mathrm{Py}]=5 \times 10^{-5} \mathrm{M}$, excitation at $346 \mathrm{~nm}$. I (Fraction 5) at $77 \mathrm{~K}$ (-----) and at room temp (fluid solution). III at $77 \mathrm{~K}(----)$.

profiles in Figure 6 and the deduced results in Table III unequivocally confirm the participation of a ground state complex. The excitation spectrum of $\mathbf{I}$ depends considerably on the monitored wavelength whereas that of II is scarcely so as shown in Table III. Furthermore, the excitation spectrum of III, independent of the monitored wavelength, indicates that the excimer system consists of a single absorb- 
ing species. Similar results were obtained for other polymers in solution. The excitation spectrum for the exciplex emission is broader than that for the monomer emission as expressed by the valley/peak ratio of the excitation spectrum manifesting that there is a broad and weak absorption band overlapping the $S_{2}-S_{0}$ band of Py responsible for a part of emission in the exciplex region.

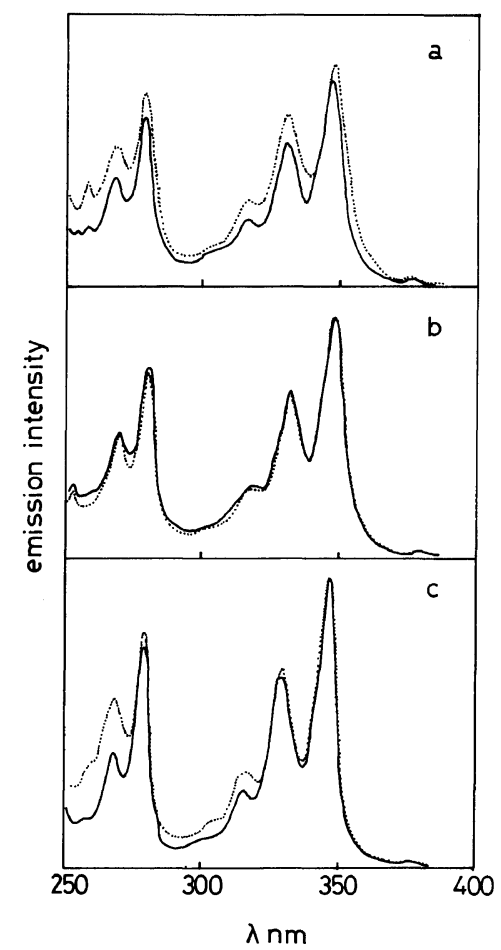

Figure 6. Excitation spectra of I (a), III (b), and II (c) in DCE. Monitored wavelength, $377 \mathrm{~nm} \mathrm{(-)}$ and $500 \mathrm{~nm}$ (-----). Absorbance $\leq 0.05$.

\section{Concentration Dependence of Exciplex Emission}

As a measure of intra- and intermolecular interactions, the intensity ratio of the exciplex emission $\left(F_{\mathrm{e}}\right)$ to that from the locally excited state of Py $\left(F_{\mathrm{m}}\right)$ was taken, and the results obtained in DCE are plotted in Figure 7. The other solvents used previously (mesitylene, $o$-xylene, dioxane, and others) are not usable because of the poor solubility of the present polymer. In the medium DP region, $(\mathrm{Fr}-5$, $\mathrm{MW}=3900$, DP $=6.5$ ), a strong concentration dependence of the exciplex formation was found and an optimum DP for the interpolymer exciplex formation was obtained. The presence of an optimum DP for the interpolymer exciplex formation was common to all exciplex forming polymers investigated. In contrast to IV, the peak in Figure 8 appears in a lower DP region and furthermore, the sharp profile of the plots indicates specific interpolymer association in a limited DP region. The difference between I and IV or VI is in part attribut-

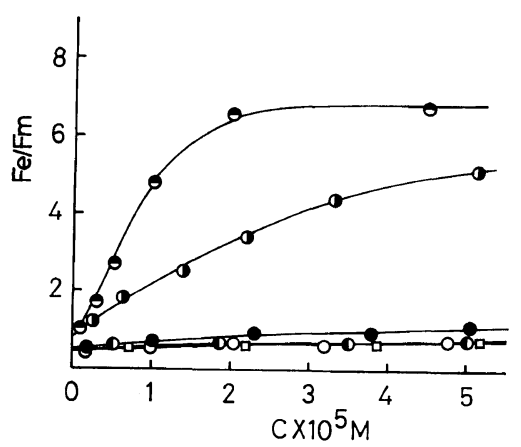

Figure 7. Concentration dependence of exciplex emission in DCE. Excitation at $346 \mathrm{~nm}$. I, fraction $9(\mathrm{O}), 7$ (D), 5 (Ө), 3 (O), and 1 (O); II, $\square$.

Table III. The valley/peak ratio of excitation spectra of I, II, and III at different monitored wavelength ${ }^{\mathrm{a}}$

\begin{tabular}{|c|c|c|c|c|c|c|}
\hline \multirow{2}{*}{$\frac{\text { Monitored wavelength }}{\mathrm{nm}}$} & \multicolumn{3}{|c|}{$F_{337} / F_{346}{ }^{\mathrm{b}}$} & \multicolumn{3}{|c|}{$F_{322} / F_{346}{ }^{\mathrm{b}}$} \\
\hline & I & II & III & I & II & III \\
\hline 377 & 0.402 & 0.331 & 0.402 & 0.298 & 0.233 & 0.281 \\
\hline 515 & 0.502 & 0.361 & 0.403 & 0.370 & 0.278 & 0.278 \\
\hline Difference $\%$ & +24.8 & +9.1 & \pm 0 & +24.2 & +19.3 & \pm 0 \\
\hline
\end{tabular}

a Conditions: The maximum OD of sample solution was maintained below $0.05\left([\mathrm{Py}]<10^{-6} \mathrm{M}\right)$. Solvent, 1,2dichloroethane. I ( $\left.\mathrm{Fr}-5, M_{w}=3900\right)$, II (urethane monomer model), and III (reference polyurethane, unfractionated $M_{w}=2000$ ).

b Suffixes indicate excitation wavelength in $\mathrm{nm}$. 


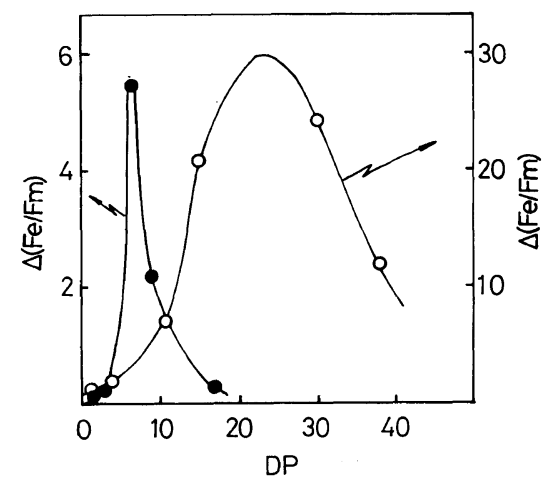

Figure 8. DP dependence of interpolymer interaction in DCE. $\Delta\left(F_{\mathrm{e}} / F_{\mathrm{m}}\right)=\left(F_{\mathrm{e}} / F_{\mathrm{m}}\right)_{2 \times 10^{-5} \mathrm{~S}}-\left(F_{\mathrm{e}} / F_{\mathrm{m}}\right)_{c \rightarrow 0} . \mathbf{I},(\mathbf{O})$; IV, (O).

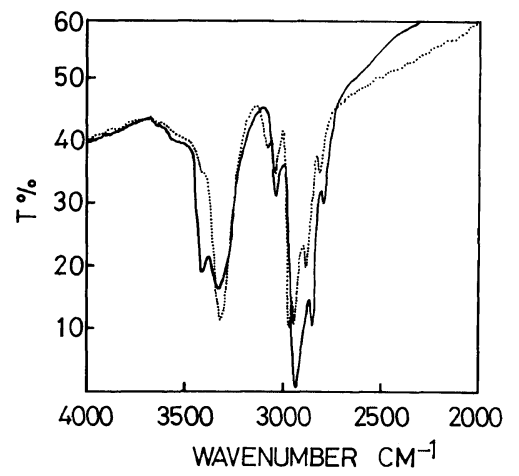

Figure 9. Infrared absorption spectra of I and II. I, (一); II, (-----).

able to the poorer solubility of I than IV and VI. When the same plots as in Figure 8 were depicted for IV and VI (ref 1, Figure 8 and ref 7, Figure 12) in poor solvents, the optimum DP value decreased and the plots became sharper but not as much as those for I in Figure 8. The hydrogen bonding tendency of the polyurethane is relevant to the difference between I and IV. The infrared absorption spectrum of I (Figure 9) provides explicit evidence for hydrogen bonding which results not only in polymer interactions but also brings about a stiff main chain. The amount of hydrogen bonding in I and III is considerably larger than that in II. The intensity ratios of the IR absorption at $3420 \mathrm{~cm}^{-1}$ (hydrogen bonded $\mathrm{NH}$ groups) to that at $3320 \mathrm{~cm}^{-1}$ (free NH groups) are 0.87 and 0.78 for I and III, respectively, whereas the value is 0.3 for II for which the absorption at $3420 \mathrm{~cm}^{-1}$ is observed only as a shoulder. If a rod-like polymer chain begins to bend above a critical DP region, polymer association may be difficult above the threshold value whereas the cooperative effect in interpolymer association ${ }^{12}$

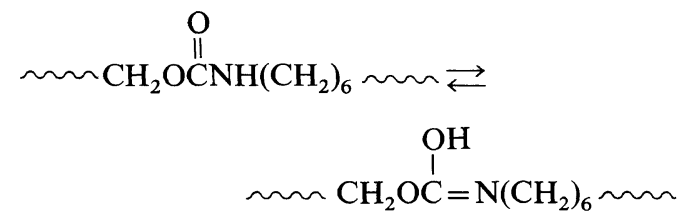

is lacking in very low DP polymers. Hydrogen bonding is however not direclty responsible for the interpolymer association. Had this been the case, III should have exhibited the interpolymer excimer formation. No such tendency was observed. ${ }^{2}$

The role of hydrogen bonding is therefore to impart rigidity to the main chain. This does not necessarily require interpolymer hydrogen bonding. Intrapolymer interaction involving the tautomeric structure shown above is possible.

The rigid and extended polymer chain of I seems relevant to the nearly DP independent intrapolymer exciplex intensity $\left(\left(F_{\mathrm{e}} / F_{\mathrm{m}}\right)_{c \rightarrow 0}\right.$ in Figure 7 and Table II). Non-neighboring group participation is not permitted for $\mathbf{I}$ at least in the present DP region. In contrast, IV showed strongly DP dependent intrapolymer exciplex in poor solvents but not in a good solvent (DCE) ${ }^{1}$ in which the polymer chain is extended. This is a reasonable consequence supported by the previous results on the DP independent intrapolymer exciplex by poly[oxy-2-(1pyrenylmethyl)propyleneoxy-(4- $N, N$-dimethylaminobenzyl)malonyl] (VI) having bulky congested side groups. ${ }^{8}$

The maximum $F_{\mathrm{e}} / F_{\mathrm{m}}$ value in Figure 7 is about 6.5 , which is much smaller than the corresponding value for IV exceeding $25 .{ }^{1}$ The energy level of the interpolymer exciplex in $\mathbf{I}$ is higher than that in IV by at least $400 \mathrm{~cm}^{-1}$. Such differences can be explained by hydrogen bonding effects on exciplex as discussed in the following section.

A red shift of up to $20 \mathrm{~nm}$ in the exciplex peaking wavelength is observed for I by increasing concentration (Figure 4 and Table II) as a reflection of the wavelength difference between the intra- and interpolymer exciplex emissions. A similar trend was reported for IV in DCE, ${ }^{1}$ but not in poor solvents. 


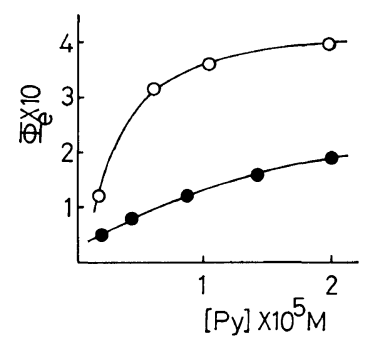

Figure 10. Quantum efficiency of exciplex emission as a function of concentration in DCE. Excitation at $346 \mathrm{~nm}$. (O), I; (O), IV.

\section{Hydrogen Bonding Effects on Exciplex Emission}

We have shown that hydrogen bonding influenced the exciplex characteristics of $\mathbf{I}$ indirectly via affecting the polymer chain rigidity. Another possibility is the effect on exciplex energy levels. In the preceding section, we considered the hydrogen bonding between carbonyl and imino groups. The DMA group is equally a hydrogen acceptor. Its basicity is higher than the carbamoyl group. The hydrogen bonding to DMA reduces the donor property and the resultant decrement in exciplex forming capability is observed. This is actually the case in Figure 8. Furthermore, the blue shifted exciplex emission by I (i.e., unstable exciplex) is consistent with this interpretation. The quantum efficiency of exciplex emission $\left(\phi_{\mathrm{e}}\right)$ is very much increased with increasing concentration. This provides direct support for the interpolymer association which promotes the exciplex formation. ${ }^{6} \phi_{\mathrm{e}}$ of $\mathbf{I}$ is considerably smaller than that of IV in all concentration regions as shown in Figure 10. This is also reminescent of the reduced donor property of DMA in $\mathbf{I}$.

NMR spectroscopy of the methyl group in DMA provides good support for the participation of hydrogen bonding. The singlet NMR signal for the methyl groups in DMA appears at 2.91 for $\mathbf{I}, 2.81$ for IV, and 2.95 for II ( $\delta, \mathrm{ppm})$. The small but considerable down field shift of I and II indicates reduced electron density owing to hydrogen bonding.

\section{CONCLUSION}

A small difference in polymer main chain architecture alters the overall picture of inter- and intrapolymer exciplex formation and the emission properties. The major effect of introducing urethane linkages to the main chain on exciplex emission is explainable by the change in main chain rigidity. However, the direct effect of hydrogen bonding on the donor-acceptor properties of DMA should also be taken into account.

\section{REFERENCES}

1. H. L. Yuan and S. Tazuke, Polym. J., 15, 111 (1983).

2. S. Tazuke and H. L. Yuan, J. Phys. Chem., 86, 1250 (1982).

3. S. Tazuke and N. Hayashi, J. Polym. Sci., Polym. Chem. Ed., 16, 2729 (1978).

4. S. Tazuke and T. Tanabe, Macromolecules, 12, 848 (1979).

5. N. Mataga and M. Ottolenghi, "Molecular Association," R. Foster, Ed., Academic Press, New York, 1979, p 24.

6. S. Tazuke and H. L. Yuan, Chem. Phys. Lett., 92, 81 (1982).

7. M. Imoto and K. Uno, "Addition Polymerization and Addition Polycondensation," Kagaku-Dojin, Kyoto, 1972, p 61.

8. S. Tazuke and H. L. Yuan, Polym. J., 14, 215 (1982).

9. S. Tazuke and H. L. Yuan, Polym. J., 14, 695 (1982).

10. H. L. Yuan and S. Tazuke, Preprints, Symposium on photochemistry, Japan, 1981, p 223.

11. Y. Iwaya and S. Tazuke, unpublished data.

12. S. Tazuke, H. L. Yuan, K. Sato, and Y. Iwaya, Macromolecules, 14, 267 (1981). 